\title{
Comparison of Uplink SAR Values in Train Environment for Different Wireless Technologies
}

\author{
D. Plets, S. Aerts, K. Vanhecke, W. Joseph, L. Martens \\ Information Technology Department, Ghent University/iMinds \\ Gaston Crommenlaan 8, B-9050 Ghent, Belgium \\ david.plets@intec.ugent.be
}

\begin{abstract}
A comparison of uplink Specific Absorption Rate (SAR) values in a train environment for different wireless technologies using macrocells or femtocells is presented. The comparison is based on both simulations and real-life experiments. Compared to a typical GSM900 macrocell scenario, the largest SAR reduction is observed when using a UMTS femtocell base station, which leads to uplink SAR reductions by a factor $\mathbf{3 5 8 , 8 2 0}$.
\end{abstract}

\section{INTRODUCTION}

The increased concerns about the possible health effects of radio-frequency (RF) electromagnetic fields have boosted the research on the characterisation of electromagnetic fields. Recently, more attention has been given to human exposure induced by the uplink (UL) traffic of wireless devices, since in areas with a bad wireless connection, exposure due to UL traffic mostly dominates exposure due to downlink (DL) traffic originating from the base stations (BS). In this paper, exposure of humans in a train environment is investigated: this is a typical environment where wireless connections are bad. A comparison is presented of UL SAR values in macro- and femtocell scenarios for GSM900 and GSM1800, and in femtocell scenarios for WiFi, Universal Mobile Telecommunications System (UMTS), and Long-Term Evolution (LTE).

\section{CONFIGURATION AND METHOD}

Seven train scenarios are investigated, for which a $20 \mathrm{~m}$ by $2.83 \mathrm{~m}$ train wagon with 66 passenger seats is considered (see Fig. 1). The uplink SAR [W/kg] at each of the 66 seats for each of the scenarios is calculated as follows.

$$
\mathrm{SAR}_{\mathrm{UL}}=\mathrm{P}^{\mathrm{Tx}} \cdot \mathrm{DC} \cdot \mathrm{SAR}_{\mathrm{REF}}^{\mathrm{UL}},
$$

where $\mathrm{P}^{\mathrm{Tx}}[\mathrm{W}]$ is the mobile device's power transmitted towards the $\mathrm{BS}$ it is connected to (MBS or FBS), DC [-] is the duty cycle of the UL traffic, and $\mathrm{SAR}_{\mathrm{REF}}^{\mathrm{UL}}[\mathrm{W} / \mathrm{kg}$ per $\mathrm{W}]$ is the reference SAR (for $1 \mathrm{~W}$ of transmitted power) due to the mobile device. For UMTS and LTE, the duty cycle is $100 \%$. Table I lists the 7 scenarios that will be considered, in which the mobile device will either connect to a GSM900 macrocell base station (MBS), to a GSM900 femtocell base station (FBS), a GSM1800 MBS, a GSM1800 FBS, a WiFi access point (AP), a UMTS FBS, or an LTE FBS. For the five scenarios involving an indoor base station (all but the two MBS scenarios), the FBS is installed at the location of the purple hexagon in Fig 1.
Table I also lists the reference $\mathrm{SAR}_{\mathrm{REF}}^{\mathrm{UL}}$ values that are used for the simulations. These uplink reference SAR values have been obtained from Finite-Difference Time-Domain (FDTD) simulations for WiFi and LTE. In the FDTD simulations, the mobile device is held in front of the body. For the other technologies, reference SAR values are obtained from [1], where the device is held to the right side of the human head. Further, Table I also lists the assumed mobile phone transmitted power values $\mathrm{P}^{\mathrm{Tx}}$. The MBS values are median values recorded along an actual railway trajectory in Belgium [2] and are assumed the same for all locations in the train wagon. The GSM1800 FBS values are also obtained from [2]. Thanks to the use of a FBS, the mobile device is able to always transmit at the lowest possible power $(0 \mathrm{dBm})$, irrespective of the location in the wagon. It is assumed that this will also be the case for the GSM900 FBS scenario (lowest transmit power $=5 \mathrm{dBm}$ ). For WiFi, a fixed transmit power of $20 \mathrm{dBm}$ is assumed, whereas for the UMTS FBS and LTE FBS scenarios, the user will fully benefit from the power control mechanism. The device transmit power for UMTS and LTE is calculated according to [3]. The path loss (PL) between the device and the FBS is calculated according to [4]. All SAR simulations in the train wagon are executed with the WiCa Heuristic Indoor Propagation Prediction (WHIPP) tool, a set of heuristic planning algorithms, experimentally validated for network planning and exposure calculations in indoor environments [3]. It allows simulating indoor wireless network deployments for different technologies and configurations (path loss model, receiver, ...).

TABLE I

Assumed SAR $\mathrm{REF}^{\mathrm{UL}}, \mathrm{P}^{\mathrm{Tx}}$, AND DUTY CYCLE (DC) VALUES FOR THE SIMULATIONS.

\begin{tabular}{|c|c|c|c|}
\hline Scenario & $\begin{array}{r}\text { SAR }_{\mathrm{REF}}^{\mathrm{UL}} \\
(\mathrm{mW} / \mathrm{kg} \text { per W) }\end{array}$ & $\begin{array}{r}\mathrm{P}^{\mathrm{Tx}} \\
(\mathrm{dBm})\end{array}$ & $\overline{\mathrm{DC}}$ \\
\hline \multicolumn{4}{|c|}{ Experiments } \\
\hline (1) GSM900 MBS & $3.85[1]$ & $21[2]$ & $1 / 8$ \\
\hline (2) GSM900 FBS & $3.85[1]$ & 5 & $1 / 8$ \\
\hline (3) GSM1800 MBS & $4.99[1]$ & $18[2]$ & $1 / 8$ \\
\hline (4) GSM1800 FBS & $4.99[1]$ & $0[2]$ & $1 / 8$ \\
\hline \multicolumn{4}{|c|}{ Simulations } \\
\hline (5) $\mathrm{WiFi}$ & 7.00 & $20[3]$ & $2 \%[3]$ \\
\hline (6) UMTS FBS & $4.95[1]$ & $-110+\mathrm{PL}[3]$ & - \\
\hline (7) LTE FBS & 7.00 & $-76+\mathrm{PL}[3]$ & - \\
\hline
\end{tabular}




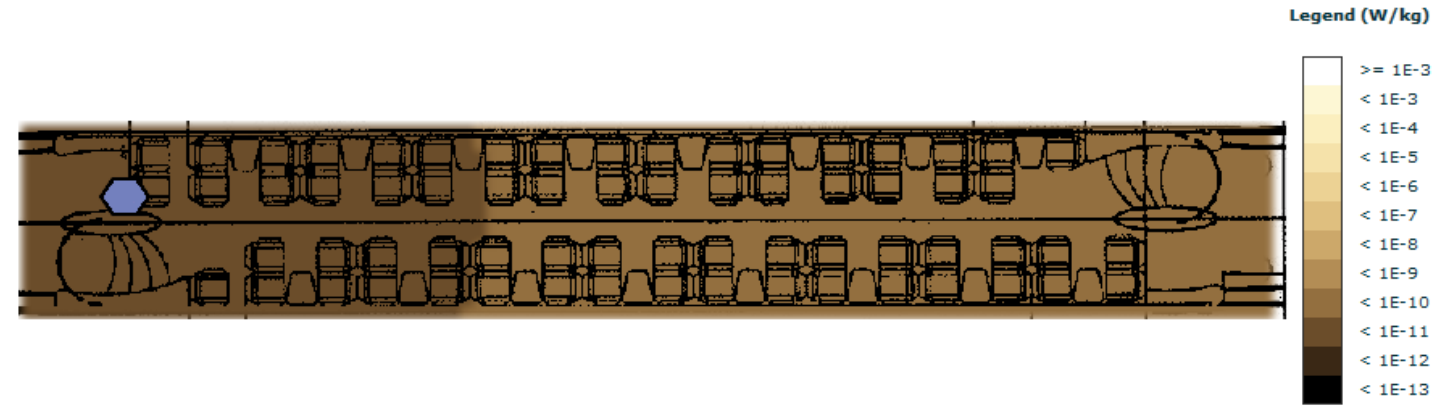

Fig. 1. Distribution of whole-body SAR due to UL traffic of the mobile device in UMTS femtocell scenario in train wagon (20 m x $2.83 \mathrm{~m})$ (UMTS FBS = purple hexagon).

\section{RESULTS}

Fig. 2 shows the cumulative distributions function (cdf) of the UL SAR for all 7 scenarios. As expected, the highest SAR values are obtained for the macrocell scenarios. The median SAR values are $61 \mu \mathrm{W} / \mathrm{kg}$ for the GSM $900 \mathrm{MBS}$ scenario, $56 \%$ higher than the value of $39 \mu \mathrm{W} / \mathrm{kg}$ for the GSM 1800 MBS scenario. The FBS scenarios yield lower median SAR values compared to the corresponding MBS scenarios. When using an FBS, the SAR reduces to $1.5 \mu \mathrm{W} / \mathrm{kg}(-97.5 \%)$ for GSM 900 and to $0.62 \mu \mathrm{W} / \mathrm{kg}(-98.4 \%)$ for GSM 1800 . The WiFi SAR values $(14 \mu \mathrm{W} / \mathrm{kg})$ are in between the GSM MBS and FBS scenarios, but WiFi allows much higher data rates. The two scenarios ((6) UMTS FBS and (7) LTE FBS, see Table I) involving advanced power control correspond with the lowest SAR values. The median SAR value for LTE is $0.18 \mu \mathrm{W} / \mathrm{kg}$, the $95 \%$ percentile is $0.48 \mu \mathrm{W} / \mathrm{kg}$. The lowest SAR values are recorded for an UMTS phone call: a median of $1.7 \cdot 10^{-5} \mu \mathrm{W} / \mathrm{kg}$ and a $95 \%$ percentile of $4.1 \cdot 10^{-5} \mu \mathrm{W} / \mathrm{kg}$. Fig. 1 shows the SAR distribution in the train. Due to the power control, very low values are observed, in particular close to the FBS, where the device transmit at the lowest possible power for UMTS, i.e., $-57 \mathrm{dBm}$. Using an UMTS FBS leads to a SAR reduction by a factor 358,820 compared to the GSM900 MBS scenario. Fig. 2 shows that even for high-rate LTE traffic, the SAR values are much lower than for WiFi (a factor 78) or GSM (a factor 3 to 8 for femtocell scenarios and a factor 217 to 339 for macrocell scenarios), confirming the benefits of advanced power control mechanisms.

\section{CONCLUSIONS}

A comparison is presented of uplink whole-body SAR values in a train environment for seven different configurations. The comparison is based on both experimental data and simulations. The use of a femtocell base station allows uplink SAR reductions of at least $97.5 \%$ for GSM scenarios. Using technologies with advanced power control mechanisms, like LTE and UMTS, result in even larger SAR reductions.

\section{ACKNOWLEDGEMENT}

This paper reports work undertaken in the context of the project LEXNET. LEXNET is a project supported by the

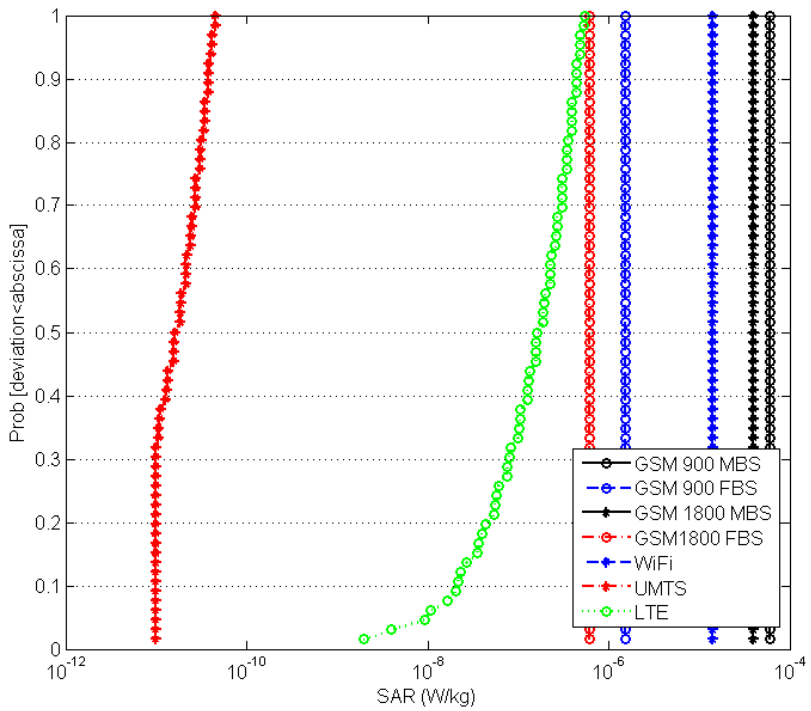

Fig. 2. Cumulative distribution function of the whole-body SAR for the seven scenarios in the train wagon of Fig. 1.

European Commission in the 7th Framework Programme (GA n318273). For further information, please visit www.lexnetproject.eu.

\section{REFERENCES}

[1] O. Lauer, P. Frei, M. Gosselin, W. Joseph, M. Roosli, and F. J., "Combining near-and far-field exposure for an organ-specific and wholebody rf-emf proxy for epidemiological research: a reference case." Bioelectromagnetics, vol. 34, no. 5, pp. 366-374, 2013.

[2] S. Aerts, D. Plets, A. Thielens, L. Martens, and W. Joseph, "Impact of a small cell on the rf-emf exposure in a train," International Journal of Environmental Research and Public Health, unpublished.

[3] D. Plets, W. Joseph, K. Vanhecke, G. Vermeeren, J. Wiart, S. Aerts, N. Varsier, and L. Martens, "Joint minimization of uplink and downlink whole-body dose in indoor wireless networks," BioMed Research International, special issue on Public Health Assessment of Impact of Heterogeneous Wireless Systems in Nonionizing Radiation Exposure, in press.

[4] Aerts S, Plets D, Verloock L, Tanghe E, Joseph W, Martens L, "Empirical path-loss model in train car," in Proceedings of the 7th European Conference on Antennas and Propagation (EuCAP), Gothenburg, Sweden, 2013, pp. 3777-3780. 